\title{
Effectiveness of external factors to reduce the dehydration risk in older people living in residential care: a systematic review
}

\author{
D. Bunn ${ }^{1}$, O. Jimoh ${ }^{1}$, S. Howard-Wilsher ${ }^{2}$ and L. Hooper ${ }^{1}$ \\ ${ }^{1}$ Norwich Medical School and ${ }^{2}$ School of Allied Health Professionals, University of East Anglia, Norwich, Norfolk. \\ NR4 7TJ, UK
}

Dehydration is prevalent amongst the elderly living in care homes ${ }^{(1)}$ because maintenance of a healthy fluid balance depends on effective physiological, mental and physical capacities, all of which are often compromised in this population. When dehydration develops, it is known to precipitate and/or exacerbate other comorbidities and result in an increased risk of hospital admissions ${ }^{(2)}$.

The aim of this systematic review was to investigate the current evidence for the effectiveness of interventions, or the impact of environmental conditions, on reducing the risk of, or reversing, impending water-loss dehydration for older people living in residential care. The review was conducted according to Cochrane Collaboration guidelines and registered with the International Register of Systematic Reviews (CRD42012003100) ${ }^{(3)}$. We included intervention or observational studies of elderly people ( $>65$ years) living in residential care, where an association was assessed between administrative, educational, behavioural, social or environmental conditions and hydration status or fluid intake. Thirteen electronic databases and registers were searched to August 2012 using index terms, subject headings, textwords and synonyms (not limited by language). We ran electronic searches of prominent authors and checked reference lists of included studies and reviews. Using predetermined criteria, two authors independently selected studies for inclusion, extracted data and assessed validity.

4164 studies were identified, after 793 duplicates were removed. 327 full text papers were obtained and 21 studies included in the review. Differences in trial design and quality, and variable methods of defining dehydration and adequate fluid intake made meta-analysis impossible.

Four US-based intervention studies with a total of 1212 residents demonstrated that increasing staff time to assist with feeding resulted in increased fluid intake, whilst one large US cross-sectional study using the Minimum Data Set $(n=363,895)$ found no relationship between staffing ratios and dehydration. A US cross-sectional study found that implementation of the Resident Assessment Instrument $(n=2128)$ was effective in reducing dehydration. A retrospective cohort study in Canada $(n=43,065)$ found that for-profit care homes had more hospital admissions for dehydration than non-profit homes. Twelve intervention studies (1 Japanese, 1 German, 10 US) investigated dehydration prevention within a care package, including increasing choice and frequency of drinks being offered and increasing toileting assistance. Two studies found no effect on hydration, 9 found positive effects, whilst one study found that although fluid intake increased, dehydration remained unchanged. Drinking receptacles were important in fluid intake, with high contrast coloured cups improving fluid intake in a US before-after study and drinking from a cup or beaker increasing fluid intake compared to use of a straw in a small UK Randomised Controlled Trial (RCT).

There are some promising interventions to improve fluid intake in care homes, but high quality well-powered RCT's are needed to confirm their efficacy.

1. Mentes J (2006) Am J Nurs 106, 40-49.

2. Davidhizar R, Dunn C \& Hart A (2004) Int Nurs Rev 51, 159-166.

3. Bunn D \& Hooper L (2012) www.crd.york.ac.uk/Prospero/display_record.asp?ID=CRD42012003100. 